\title{
An unexpected consequence of a roller coaster ride
}

\author{
K Lascelles, D Hewes, V Ganesan
}

Cervicocephalic arterial dissections are an important cause of stroke in young people with potential treatment implications. They are implicated in the aetiology of about $5 \%$ of ischaemic strokes in the paediatric age group. ${ }^{1-2}$ Recognition is often delayed because the clinical symptoms (headache and neck pain) are non-specific and the diagnosis may not be considered until there is evidence of neurological impairment. Furthermore, the diagnosis may be missed unless targeted investigations are carried out. This may adversely affect the clinical course.

\section{Case report}

An 11 year old boy was taken to an amusement park during the school holidays. Towards the end of the day, he went on a roller coaster ride. The ride came to a sudden stop and he immediately experienced a generalised headache, which lasted 1 week. He was then well enough to go to another amusement park, where he enjoyed several other rides. Over the next 2 weeks, he experienced recurrent, transient, episodes of hemiparesis (affecting both the right and the left sides) and ataxia associated with headache, dizziness, nausea, and vomiting. On presentation at hospital, he was found to have both cerebellar and pyramidal signs on the left.
He had no phenotypic features of a connective tissue disorder.

An initial brain CT showed a left cerebellar infarct. As the underlying cause was not apparent, MRI and magnetic resonance angiography (MRA) were performed. The MRI showed the previously recognised left cerebellar infarct but also showed additional infarcts in both thalami (fig 1).

In addition, the axial $\mathrm{T} 2$ weighted images showed that the lumen of the left vertebral artery was reduced in calibre compared with the right. There was concentric high signal within the wall of the left vertebral artery (fig 2) and the MRA showed absence of flow within this vessel. These appearances were diagnostic of left vertebral artery dissection. Cervical spine radiography was normal.

$\mathrm{He}$ was anticoagulated with low molecular weight heparin followed by long term warfarin. He made an excellent recovery with minimal residual impairment.

\section{Discussion}

Neurological complications after roller coaster rides have been described in 15 young adults since $1994 .^{3-4}$ These include three cases of vertebral artery dissection and five cases of internal carotid artery dissection. Initial symptoms of headache, neck pain, or both preceded

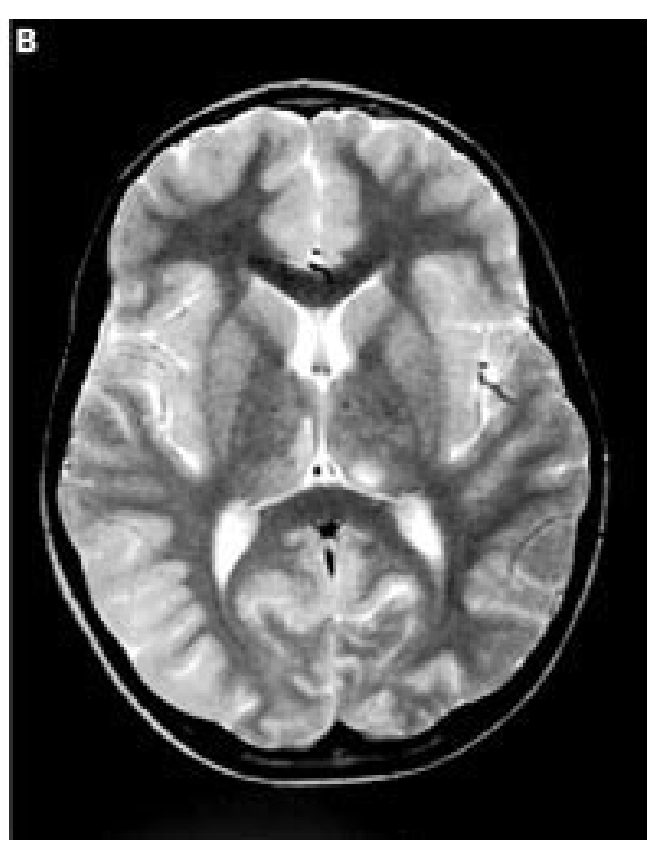

Figure 1 Axial T2 weighted MRI showing (A) Left cerebellar and (B) bilateral thalamic infarcts. 


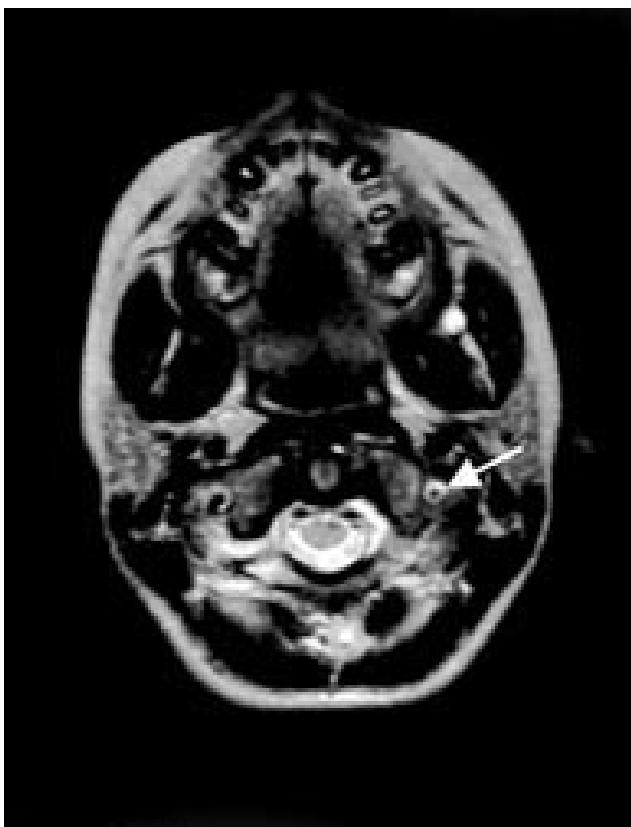

Figure 2 Axial T2 weighted MRI showing reduction in calibre of the left vertebral artery relative to right and concentric high signal within the wall of the left vertebral artery (arrow).

neurological impairment in all patients, with a delay in the onset of neurological symptoms of up to 11 days. Although there are clear warnings in amusement parks for particular groups of people such as pregnant women, those with cardiac histories, general ill health, the very young, and elderly, people, all these reported complications were unexpected. The excitement of roller coaster rides is partly derived from complex and abrupt changes in motion. This has the potential to cause sudden hyperextension, hyperflexion, or rotation of the neck, which may cause injury to the carotid or vertebral arteries. Certain people, such as those with underlying connective tissue disorders or cervical vertebral abnormalities, ${ }^{5}$ may be more susceptible to arterial dissection. However, there is evidence to suggest that patients who are phenotypically normal may have ultrastructural abnormalities of connective tissue. ${ }^{6}$ The rationale for the use of anticoagulation in patients with arterial dissection is to prevent early recurrence. There is anecdotal evidence that outcome is better if the diagnosis is made and treatment initiated before cerebral infarction has occurred. ${ }^{7}$ It is therefore important to consider the diagnosis in patients presenting with a headache after activities involving sudden neck movements-for example, chiropractic manipulation, tennis, or even routine anaesthesia. $^{8}$ If the diagnosis is suspected targeted investigations such as axial $\mathrm{T} 1$ weighted MRI with fat saturation can be used to image the neck vessels. Magnetic resonance imaging has been shown to be a sensitive, noninvasive modality for diagnosis. ${ }^{9}$ However, conventional cerebral angiography remains the gold standard and should be considered if there is a high level of clinical suspicion. ${ }^{2}$ Given that some affected patients may have an underlying susceptibility to dissection, it would seem prudent to advise all patients to avoid contact sports. However, given the wide variety of routine activities which have been associated with arterial dissection, this advice may need to be tailored to the lifestyle of the individual patient.

1 Williams LS, Garg BP, Cohen MD, et al. Subtypes of ischaemic stroke in children and Young adults. Neurology 1997; 49:1541-5

2 Ganesan V, Savvy L, Chong W, et al. Conventional cerebral angiography in children with ischaemic stroke. Pediatr Neurol 1999;200:38-42

3 Kettaneh A, Biousse V, Bousser MG. Neurological complications after roller coaster rides: An emerging risk? Presse Med 2000;29:175-80

4 Burneo JG, Shatz R, Papamitsakis NIH, et al. Amusement park stroke. Neurology 2000;55:564

5 Cushing KE, Ramesh V, Gardner-Medwin D, et al. Abberrant course of the vertebral artery through congenital arcuate foramen of the atlas vertebra: a possible cause of vertebral artery stroke in children. BPNA abstracts 1998. Dev Med Child Neurol 1997;Supplement 77:5

6 Brandt T, Haussrt I, Orberk E, et al. Ultrastructural connective tissue abnormalities in patients with spontaneous cervicocerebral artery dissections. Ann Neurol 1998;44: $281-5$

7 Sturzenegger M. Spontaneous internal carotid artery dissection: early diagnosis and management in 44 patients. dissection: early diagnosis

8 Caplan LR, Zarins CK, Hemmati M. Spontaneous dissection of the extracranial vertebral arteries. Stroke 1985;16:1030-7

9 Ozdoba C, Sturzenegger M, Schroth G. Internal carotid artery dissection: MR imaging features and clinical radiological correlation. Radiology 1996;199:191-8 